\title{
Elastic Multilayer Bandages for Chronic Venous Insufficiency: Features of Our Technique
}

\author{
Kotaro Suehiro, MD, ${ }_{1}^{1}$ Maki Okada, ${ }^{2}$ Aiko Yoshimura, ${ }^{2}$ Noriyasu Morikage, MD, ${ }^{1}$ Masanori Murakami, MD, ${ }^{1}$ \\ Osamu Yamashita, MD, ${ }^{1}$ and Kimikazu Hamano, $\mathrm{MD}^{1}$
}

\begin{abstract}
Objectives: To evaluate the interface pressure (IP) and stiffness of our elastic multilayer bandages (eMLB). Methods: Three medical staff wrapped the legs of $\mathbf{1 0}$ healthy volunteers with one to six rolls of elastic bandages. The IP was measured at the medial aspect of the lower leg at the level of transposition of the medial gastrocnemius muscle into the Achilles tendon (level of B1) with the patient supine and then standing, for each number of bandages worn. The static stiffness index (SSI) was calculated as a difference between these IPs.

Results: The IPs in the standing position increased linearly for up to five bandages $(21.8 \pm 7.2,32.5 \pm 6.1$, $41.8 \pm 8.5,52.0 \pm 10.4,60.3 \pm 11.8$, and $66.7 \pm 13.4 \mathrm{mmHg}$, with one to six bandages). SSI also increased linearly for up to five bandages $(6.8 \pm 5.1,10.2 \pm 4.8,13.4 \pm 7.2,17.4 \pm 8.8,19.7 \pm 9.1$, and $20.4 \pm 9.4$ mmHg, with one to six bandages). No significant technical variation in the IP was observed among the three operators.
\end{abstract}

Conclusions: Our eMLB provided stable, predictable and sufficient IPs and SSIs in healthy volunteers.

Keywords: compression therapy, elastic bandage, chronic venous insufficiency

\section{INTRODUCTION}

$\mathrm{C}$ ompression therapy is the primary treatment for chronic venous insufficiency (CVI) and is generally provided by compression stockings or bandages. Various methods and materials are available for compression therapy, none of which has been proven to be superior to another. ${ }^{1)}$

Stable and predictable interface pressure (IP) can be achieved by stockings; therefore, physicians are com-

${ }^{1}$ Division of Vascular Surgery, Department of Surgery and Clinical Science, Yamaguchi University Graduate School of Medicine, Ube, Yamaguchi, Japan

${ }^{2}$ Department of Nursing, Yamaguchi University Hospital, Ube, Yamaguchi, Japan

Received: February 24, 2012; Accepted: June 14, 2012 Corresponding author: Kotaro Suehiro, MD. Division of Vascular Surgery, Department of Surgery and Clinical Science, Yamaguchi University Graduate School of Medicine, 1-1-1, Minamikogushi, Ube, Yamaguchi 755-8505, Japan

Tel: +81-836-22-2260, Fax: +81-836-22-2423

E-mail: ksuehiro-circ@umin.ac.jp fortable to prescribe them for patients to use. However, pulling up thick, tight stockings is difficult, particularly for weak, elderly, and handicapped patients, and for these patients, bandages may be a better choice. Although bulky, compression bandages can be applied to legs of any shape, with or without dressings inside. One of the major disadvantages of compression achieved using bandages is unpredictable IPs, mainly because of the individual variations of tensions when applied. To overcome this problem, we started to instruct patients to apply elastic bandages, not by stretching them, but by just putting them on the leg and then smoothing them out. This technique was so compliant that most patients and/or their caregivers could master it with minimal training.

We introduce our technique of applying elastic multilayer bandages (eMLB) designed to treat CVI. We also describe their compression characteristics, measured by the IP and stiffness achieved, derived from our eMLB. 



Fig. 1 Bandaging technique.

Elastic bandages are applied to fit around the leg, but without excessive tension. This can be achieved by laying the bandage softly on the leg and smoothing out the wrinkles in a bandage in an advancing direction (A). These bandages should cover the area between the popliteus and the ankle evenly (B).

\section{Patients and Methods}

This study was approved by Institutional Review Board of Yamaguchi University Hospital (Ube, Yamaguchi, Japan). All participants signed an informed consent before enrollment in this study. The subjects were 10 healthy volunteers, comprising two men and eight women, aged 23-50 (median 29) years, whose legs were wrapped in bandages by three different medical staff. We used Getto elastic bandage ${ }^{\circledR} 10 \mathrm{~cm} \times 4.5 \mathrm{~m}$ (Kawamoto corporation, Osaka, Japan), which is made of $100 \%$ cotton and is relatively soft and thin so that even someone inexperienced can handle it easily. The extensibility of this bandage is reported to be $108 \%$, making it an elastic (long-stretch) bandage.

The most distinct characteristic of our technique is the elimination of the intentional stretch of bandages when they are applied. In the supine position, volunteers had bandages applied between the ankle and the popliteus, just to fit the leg but without stretching them intentionally. This can be achieved by placing the bandage softly around the leg and smoothing out the wrinkles in it in an advancing direction (Fig. 1A). By using this method, the bandage stays in its place and does not loosen. The bandages were put on the leg evenly in a spiral way, beginning at the ankle and moving upward to the popliteal fossa, to cover the whole calf muscle (Fig. 1B). In a practical situation, the wrapping is generally started from the foot. However, in order to eliminate the technical variation and to simplify the technique, the wrapping of the foot was omitted in the current study. The second and subsequent bandages were applied over and over in the same way. In the current study, we wrapped the lower leg with one to six rolls of bandages, in order.

IP was measured using an air pack-type analyser (Model AMI-3037-SB, AMI Co., Tokyo, Japan). This was attached to the medial aspect of the lower leg at the level of transposition of the medial gastrocnemius muscle into the Achilles tendon, at the level of B1..2) The IP was measured first with the volunteer in the supine position and then in the standing position, after each of the six bandages was applied. All the measurements were performed between 3 p.m. and 5 p.m.

We measured the stiffness of bandages using the static stiffness index (SSI). The SSI is defined as the difference between IPs in the supine and standing position $(\mathrm{mmHg}){ }^{3)}$

\section{Statistical Analysis}

Results are expressed as means \pm standard deviation, unless otherwise indicated. The Mann-Whitney $U$-test was used to calculate the significances in overall changes in IPs and SSIs. The Kruskal-Wallis test was used to 


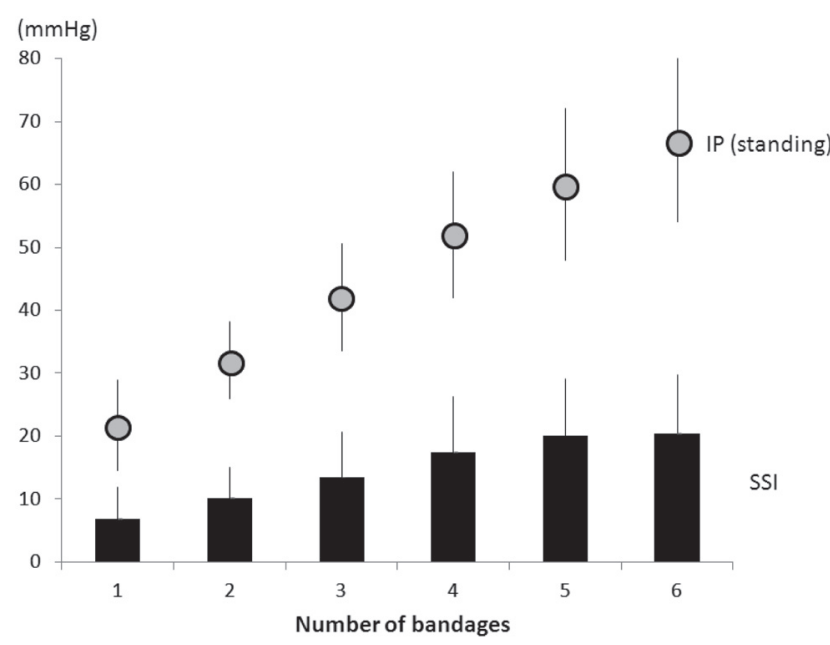

Fig. 2 Interface pressures and static stiffness indices derived from the multilayer bandages.

IP: interface pressure; SSI: static stiffness index

compare IPs obtained by the three different people who applied the bandages. Statistical analyses were performed using StatView J-5.0 (SAS Institute, Cary, NC, USA). A $p$-value of less than 0.05 was considered significant.

\section{Results}

The leg length (the distance between the head of the fibula and the upper edge of the lateral malleolus), the circumference of the ankle, and the maximum circumference of the calf were $31.3 \pm 3.7,20.9 \pm 1.6$, and $36.0 \pm$ $3.2 \mathrm{~cm}$, respectively, in the ten volunteers.

Figure 2 shows the overall changes in IPs of the standing position and the SSIs according to the number of bandages applied to the leg in the entire group. The IP of the standing position was $21.8 \pm 7.2 \mathrm{mmHg}$ when the leg was wrapped using only one roll of bandage. By the addition of each roll of bandage, the IP of the standing position increased significantly to $32.5 \pm 6.1,41.8 \pm 8.5$, $52.0 \pm 10.4,60.3 \pm 11.8$, and $66.7 \pm 13.4 \mathrm{mmHg}$, with two to six rolls, in this order. The increase in IP seemed to be roughly linear. The SSI also increased significantly with each bandage, except for bandage no. 6 ; to $6.8 \pm 5.1$, $10.2 \pm 4.8,13.4 \pm 7.2,17.4 \pm 8.8,19.7 \pm 9.1$, and $20.4 \pm 9.4$ $\mathrm{mmHg}$ with one to six rolls, respectively, in this order. Again, the increase seemed to be linear for up to five rolls, but this linear increase in SSI was not observed between five and six rolls. No significant technical variation was observed among the three operators (Fig. 3).



Fig. 3 Interface pressures achieved by multilayer bandages applied by three different people.

\section{Discussion}

The findings of the present study demonstrated that IPs and SSIs obtained by our eMLB technique changed linearly with the number of bandages applied, for up to five rolls. Moreover, similar IPs and SSIs could be achieved by three different people applying the bandages.

The number of elderly patients with CVI, who cannot wear appropriate compression stockings is increasing. Although compression therapy using bandages is indicated for these patients, they often cannot bend forward enough, and find it difficult to bandage around the ankle with effective pressure. Patients with impaired use of their hands, such as those with rheumatoid arthritis, and those who have suffered cerebral infarction have similar problems. Out of necessity, we started to tell them to apply bandages softly over the skin and smooth out the wrinkles in the bandage in an advancing direction without thinking of "tension," but this technique was proved to generate stable IPs in the current study. Generally, bandages are continuously stretched with constant tension, but it is difficult to achieve stable IPs in this way. Stretching bandages to the same degree over and over again is difficult, even for an experienced person. Furthermore, the degree of stretch is hard to explain to the patients and/or their caregivers. One may wrap the leg with all their strength, but another may apply bandages loosely. Variable tension causes unpredictable IP under bandages, which results in an uncontrollable compression therapy. We tell patients to apply bandages like "pasting" or "smoothing out." This technique is very achievable for everyone, so almost all 
patients are able to master this technique within 1 week, and usually within 2-3 days, as long as they can reach their feet with either hand. Bandages can also be kept in place easily by using this technique.

Since the current Japanese support system has not been prepared well for these weak patients, they cannot constantly find proper bandagers, and they eventually fall off from the treatment. Therefore, we would like to emphasize that the feasibility of "self-bandaging" is quite important to keep the treatment of patients on course. As described above, our technique can be used as long as the patients can reach their feet with either hand, i.e., just one hand. For instance, one of our patients with a paralyzed right hand due to a cerebral infarction can wrap both legs with only his left hand,using our technique. Another patient with fixed fingers due to rheumatoid arthritis also can wrap her legs. Most of our big fat patients with BMI $>40$ can wrap their legs using this technique as the application of bandages is easy. Of course, it is still a hard work for them to apply many bandages, but with this technique, they can do it by themselves.

In the current study, the inter-operator variation in IP was small with this technique. Actually, we measure sub-bandage pressures with eMLB done by patients themselves in the clinic, which concur with the present results.

The proper application of inelastic bandages is technically demanding, particularly for these patients; therefore, we recommend that they use elastic bandages, which have practical benefits over inelastic bandages. Moreover, patients with CVI should wear compression stockings or bandages for years and often for life. However, in patients with venous ulcers, bandages are frequently contaminated by exudates from the wound and have to be discarded and replaced regularly. As the cost of these bandages is not covered by insurance in Japan, bandages should be inexpensive. The bandage used in the current study costs approximately $500 \mathrm{yen} / \mathrm{roll}$. Since four to five rolls are practically used to wrap one leg, it would cost 2000-2500 yen per leg. Another benefit of elastic bandages is stability, in terms of the IP. Larsen et al. ${ }^{4}$ reported that the working pressure at ankle level 7-8 hours after the application of inelastic bandages dropped to approximately $75 \%$ of the initial resting pressure, whereas, the pressure after the application of elastic bandages dropped only to $95 \%$. Of course, this problem is resolved by frequent reapplication of the bandages, but it is certainly a hard job for weak patients.

In the supine position, an external pressure of 10-15 $\mathrm{mmHg}$ is enough to constrict the venous diameter, which increases the blood flow in the deep veins. ${ }^{5}$ In the upright position, the venous pump is improved by reducing venous reflux, first evident by an ankle pressure of around $20 \mathrm{mmHg}$. Elastic bandages reduce the venous reflux significantly, achieving an ankle pressure of $40 \mathrm{mmHg} .{ }^{6}$ ) In the clinical setting, it is generally recommended that the IP in the upright position is $30-40 \mathrm{mmHg}$ for CEAP classes 4 or greater, and 40 to $50 \mathrm{mmHg}$ for recurrent ulcers. ${ }^{7)}$ However, it has also been reported that a higher healing rate of venous leg ulcers could be achieved with even higher IPs. ${ }^{8)}$

In the current study, the IP in the standing position, achieved by one elastic bandage, was $21.8 \pm 7.2 \mathrm{mmHg}$, which is of course suboptimal to treat CVI. Accordingly, to increase the sub-bandage pressure and stiffness of wrapping, the bandages were laid one on top of another. Based on our observation of patients in the clinic, the eMLB achieved by using four elastic bandages on each leg improved the various CVI symptoms in almost all cases. The IPs and SSIs achieved by our eMLB technique increased almost linearly in accordance with the number of bandages applied. To achieve an IP of $40 \mathrm{mmHg}$ in the standing position, which would treat CVI, three bandages applied between the ankle and the popliteus seems appropriate. Since most patients start to apply bandages from the foot, to prevent pedal edema, they would require one more bandage, concurrent with our clinical experience.

As the IP achieved by using this bandage technique is predictable, for an average leg, adding one more bandage will increase the IP by approximately $10 \mathrm{mmHg}$. Knowing this, the control is simple: just add one more bandage if the symptoms do not improve, to increase the IP from 40 to $50 \mathrm{mmHg}$. Since the addition of a sixth bandage did not seem to increase the IP and SSI, and as the application of so many bandages would not be desirable, inelastic bandages may be considered for severe cases. Variations of this technique may also be developed. For instance, adding one elastic bandage will change a class II stocking to a class III stocking. As a matter of course, the IP should be measured regularly so that the disorder is well controlled.

The overlapping of bandages increases friction and stiffness. Our findings showed that the SSI increased with the number of bandages. The SSI derived from our eMLB by using three bandages was $13.4 \pm 7.2 \mathrm{mmHg}$, which is comparable to that achieved by short-stretch bandages $\left(\right.$ Complilan $\left.^{\circledR}\right)$, as reported by Hirai et al. $\left.{ }^{9}\right)$ Milic et al. also suggested that a higher venous ulcer healing rate was achieved with an SSI greater than $10 \mathrm{mmHg} .{ }^{8)}$ 
This again supports the effectiveness of our eMLB using three or more bandages to treat CVI.

\section{Limitations}

In the presented study, only IPs measured immediately after application of bandages in healthy volunteers were discussed. However, IP in the static condition alone cannot be a surrogate parameter for the efficacy of compression bandages, but it should be evaluated in a dynamic state. Moreover, the study should be performed in patients with CVI who have applied the bandages by themselves in order to elucidate the efficacy of this technique in the clinical setting.

\section{Conclusion}

Our eMLB technique achieved stable and predictable IPs and SSIs, when it is done by technicians. Taking our clinical experiences into consideration, the technique may be beneficial not only for weak, elderly, and handicapped patients, but also for any patient with CVI who needs compression bandages.

\section{Disclosure Statement}

There are no conflicts of interest to declare.

\section{REFERENCES}

1) Gloviczki P. Handbook of venous disorders: guidelines of the American Venous Forum. Third edition. ed: Oxford University Press 2009.

2) Partsch H, Clark M, Bassez S, et al. Measurement of lower leg compression in vivo: recommendations for the performance of measurements of interface pressure and stiffness: consensus statement. Dermatol Surg 2006; 32: 224-32, discussion 233. [Medline] [CrossRef]

3) Partsch H. The static stiffness index: a simple method to assess the elastic property of compression material in vivo. Dermatol Surg 2005; 31: 625-30. [Medline] [CrossRef]

4) Larsen AM, Futtrup I. Watch the pressure-It drops. EWMA journal. 2004; 4: 8-12.

5) Partsch B, Partsch H. Calf compression pressure required to achieve venous closure from supine to standing positions. J Vasc Surg 2005; 42: 734-8. [Medline] [CrossRef]

6) Partsch H, Menzinger G, Mostbeck A. Inelastic leg compression is more effective to reduce deep venous refluxes than elastic bandages. Dermatol Surg 1999; 25: 695-700. [Medline] [CrossRef]

7) Eberhardt RT, Raffetto JD. Chronic venous insufficiency. Circulation 2005; 111: 2398-409. [Medline] [CrossRef]

8) Milic DJ, Zivic SS, Bogdanovic DC, et al. The influence of different sub-bandage pressure values on venous leg ulcers healing when treated with compression therapy. J Vasc Surg 2010; 51: 655-61. [Medline] [CrossRef]

9) Hirai M, Niimi K, Iwata H, et al. A comparison of interface pressure and stiffness between elastic stockings and bandages. Phlebology 2009; 24: 120-4. [Medline] [CrossRef] 